\title{
Modelo de valoración de riesgo financiero en la gestión de contratos de suministro de energía eléctrica
}

\section{Financial-risk assessment model for electrical-energy supply bilateral contract management}

\author{
Mónica SÁNChez \\ Ingeniera Industrial, magíster en Ingeniería Eléctrica. Ingeniera de EPSA. Cali, \\ Colombia. Contacto: msanchez@epsa.com.co \\ Carlos A. Lozano \\ Ingeniero Electricista, magíster en Ingeniería Eléctrica. Docente de la Universi- \\ dad del Valle. Cali, Colombia. Contacto: carlos.a.lozano@correounivalle.edu.co \\ Diego Manotas \\ Ingeniero Industrial, magíster en Ingeniería Industrial. Docente de la Universi- \\ dad del Valle.Cali, Colombia. Contacto:diego.manotas@correounivalle.edu.co \\ Fecha de recepción: 11 de septiembre de 2012 \\ Clasificación del artículo: investigación \\ Fecha de aceptación: 27 de agosto de 2013 \\ Financiamiento: Universidad del Valle - EPSA
}

Palabras clave: valor en riesgo (VaR), valor en riesgo condicional (CVaR), mercados de electricidad, contratos bilaterales.

Keywords: value at risk (VaR), conditional value at risk (CVaR), electricity markets, bilateral contracts.

\section{RESUMEN}

Este artículo presenta la valoración del riesgo financiero en el proceso de venta de energía eléctrica mediante contratos a largo plazo. La volatilidad que exhiben los precios spot de la bolsa de energía en Colombia es uno de los aspectos de mayor incidencia en la cuantificación del riesgo financiero en el sector eléctrico, donde las con- diciones climatológicas son determinantes fundamentales del precio en un país cuya fuente de generación principal es la hidroelectricidad.

En este artículo se introduce un caso aplicado desde la perspectiva de uno de los agentes del mercado eléctrico, el generador. El modelo se desarrolló mediante la definición de distribuciones de probabilidad para las variables de entrada, 
la aplicación del método de simulación Monte Carlo y el análisis de indicadores robustos de medición de riesgo como el valor en riesgo VaR (Value at Risk) y el valor en riesgo condicional CvaR (ConditionalValue at Risk), en un horizonte de tiempo dado y considerando distintos escenarios, lo cual brinda argumentos cuantitativos adecuados para un análisis más detallado en el proceso de toma de decisiones. Una vez analizadas las series históricas de precios de bolsa y de contratos, la ejecución del análisis de escenarios y la evaluación de los indicadores financieros, se propone un esquema de portafolio de contratación óptima (bolsa y contratos bilaterales) basado en el nivel de aversión al riesgo del generador que apoya el proceso de toma de decisión.

\section{ABSTRACT}

This paper presents a financial risk assessment model for the electrical-energy-sale process trough long-term bilateral contracts. The volatility exhibited by spot prices of electricity in Colombia constitutes one of the aspects of big influence in financial risk assessment within the electrical sector, where weather conditions are fundamental in the consolidation of electricity prices in a country whose major generation source is hydroelectricity.

Moreover, in this paper, a case study is introduced from the perspective of one of the electricity market agents, namely the generator. The model is developed by means of probability-distribution definitions that represent input variables. Monte Carlo simulation methods were applied together with the analysis of robust risk indicators VaR (Value at Risk) and CVaR (Conditional Value at Risk), over a given time horizon and also considering different scenarios. This provides proper quantitative arguments for more detailed decision-making processes. Once the historic series of spot prices and contracts are analyzed and the analysis scenario has been deployed together with its corresponding financial risk assessment, a contracting optimal portfolio scheme (spot and bilateral contracts) is proposed based on the riskaversion level of the generator that supports the decision-making process.

\section{INTRODUCCIÓN}

Como consecuencia de los procesos de reforma del mercado eléctrico desde la década del noventa y con el objetivo de incrementar la eficiencia del sector de energía eléctrica colombiano, se han implementado procesos de liberalización y desregulación, los cuales han incentivado la participación de los agentes dentro de un esquema de libre mercado.

El sistema eléctrico colombiano posee la particularidad de que las empresas comercializadoras y los grandes consumidores adquieren la energía $y$ potencia en un mercado de grandes bloques de energía, el cual opera libremente de acuerdo con las condiciones de oferta y demanda [1].

Para promover la competencia entre generadores, se permite la participación de agentes económicos, públicos y privados, los cuales deberán estar integrados al sistema interconectado para participar en el mercado de energía mayorista. Como contraparte, comercializadores y grandes consumidores actúan celebrando contratos de suministro de energía eléctrica con los generadores. El precio de la electricidad en este mercado se establece de común acuerdo entre las partes contratantes, sin la intervención del Estado [1]. 
Los nuevos mercados eléctricos competitivos hacen imprescindible que las empresas de generación cuenten con herramientas de soporte para la medición del riesgo. La predicción del nivel de riesgo y el punto óptimo de contratación resultan críticos al momento de realizar ofertas a mercados diversos, debido a que la predicción de precios de energía eléctrica es sensiblemente más compleja que la predicción de la demanda, dado que el nivel de incertidumbre es mayor. Conocer el nivel de riesgo con anterioridad hace posible que los generadores determinen su mejor estrategia de contratación, de tal forma que se optimice la relación riesgo-utilidad.

Por otra parte, consumidores y generadores de energía eléctrica preferirán tener una mayor certeza sobre los precios futuros de electricidad; la incertidumbre crea riesgos y la mayor parte de los individuos son aversos a estos. Para el consumidor, el riesgo se presenta cuando el precio de la electricidad sea más alto que el previsto, mientras que para el generador el riesgo se presenta cuando el precio es inferior al que había esperado. Dado que ambos son aversos al riesgo, el consumidor y el productor prefieren protegerse sobre variaciones de precio. En este sentido, la electricidad es igual a cualquier otro activo de la economía por lo que generadores y consumidores han desarrollado mecanismos para protegerse de variaciones de precio, principalmente por medio de contratos a mediano y largo plazo.

En la literatura sobre economía energética se reportan algunos trabajos relacionados con la utilización de técnicas de optimización de portafolio, las cuales son asimilables a la metodología que se utilizó en el presente trabajo. En [2] se presenta un modelo de portafolio para determinar la posición óptima en términos de contratos forward y contratos de opciones en el mercado spot de energía, concluyendo que la adopción de posiciones activas en contratos forward disminuye significativamente el riesgo de mercado de los procesos de venta de electricidad. Herguera [3] presentó un análisis detallado del comportamiento de los precios de la electricidad tanto en el mercado spot como en el mercado de contratos bilaterales, identificando una serie de hechos estilizados en el comportamiento de los precios de la electricidad como son agrupamientos de volatilidad, reversión a la media y estacionalidad. Ambos trabajos fueron desarrollados en los mercados eléctricos de Escandinavia y Reino Unido. Manzano [4] presenta un trabajo similar al propuesto en el presente documento aplicado al mercado brasilero, destacando la construcción de un portafolio óptimo de comercialización de energía para un generador eléctrico, el cual tiene la posibilidad de interactuar en el mercado spot y en el mercado de contratos bilaterales. Como elemento de interés se destaca el análisis de correlación que se realiza entre los precios de ambos mercados.

Es conveniente señalar que el problema de la comercialización óptima de electricidad no ha sido tratado solo desde la óptica de las compañías de generación y en general, de las compañías comercializadoras. Este problema, además, es de particular interés para grandes consumidores que deben realizar una gestión activa de sus compras de energía. En este contexto, la función objetivo cambia hacia la minimización de los costos de suministro. En [5] se presenta un modelo de optimización estocástica para optimizar el abastecimiento energético de un gran consumidor que enfrenta la posibilidad de contratar la energía en el mercado spot o vía contratos bilaterales.

Liu y $\mathrm{Wu}[6]$ presentan un modelo general de optimización de portafolios de comercialización de energía eléctrica para compañías de generación que tienen la posibilidad de interactuar tanto en el mercado spot como en el mercado de contratos bilaterales. En este trabajo se utilizan los mismos criterios de la teoría clásica de valoración de portafolio, aproximación media-varianza, mediante 
la consideración de portafolios de comercialización que incluyen una definición de activo libre de riesgo, correspondiente a la utilización de contratos y activos riesgosos, representados por los precios spot en diferentes zonas. Khatib y Galiana [7] proponen un esquema de negociación dinámico en el cual la interacción de la compañía de generación en cada mercado se va ajustando de acuerdo con el comportamiento de los precios spot y forward de la electricidad. Este modelo contempla la posibilidad de que la compañía de generación cumpla sus contratos de ventas a partir de la compra de energía en el mercado spot cuando le conviene. En [8] se presenta un modelo de optimización multi-objetivo para determinar una frontera eficiente de negociación de contratos bilaterales en mercados de energía desregulados.

Autores como Nerves y Umali [9] desarrollaron una metodología para valorar el riesgo financiero que enfrenta una compañía de generación en su proceso de venta de energía vía contratos bilaterales y mercado spot. La metodología desarrollada se basa en la estimación del valor en riesgo (VaR) calculado mediante simulación de Monte Carlo para valorar diferentes escenarios de precio.

Karandikar [10] presenta un modelo de valoración de riesgo financiero basado en la técnica conocida como RAROC (Risk Adjusted Recovery on Capital), recuperación sobre capital ajustada por riesgo. En este modelo se realiza un análisis de las posiciones de riesgo que deben enfrentar los generadores en su rol de comercialización, cuando reciben pagos fijos por parte de los consumidores. El principal resultado del modelo desarrollado es una franja de negociación para la utilización de contratos bilaterales.

En [11] se presenta una aplicación de los conceptos clásicos de valoración de portafolio en un problema de valoración del riesgo de inversión, para comparar las posiciones activas en relación con el suministro de capacidad y la generación real de energía. La aplicación desarrollada se presenta para evaluación de plantas de generación eólicas y se incluyen aspectos como los costos de arranque, alistamiento y parada. En Kebriaei [12] se presenta un modelo de simulación basado en agentes para determinar la cantidad óptima de contratación bilateral que se debe asumir en un mercado, teniendo en cuenta los conflictos que se presentan entre quienes compran y las compañías de generación, basado en el modelo de equilibrio de Stackelberg. En [13] se presenta un modelo de contratación bilateral en el mercado eléctrico colombiano basado en la utilización de técnicas de medición de riesgo financiero. El modelo desarrollado se utiliza para fijar precios base para negociación bilateral entre comercializadores y agentes generadores, dado un cierto nivel de tolerancia al riesgo por parte de los agentes.

Gökgöz [14] desarrolló un modelo de optimización financiera para definir el portafolio de contratos forward y participación en el mercado spot de energía en Turquía. La aproximación desarrollada en este trabajo recurre a la teoría clásica de media-varianza y modela los cambios en los precios de electricidad a partir de la distribución normal. En este artículo se incluyen también restricciones técnicas en las unidades de generación de la compañía.

Los desarrollos investigativos en torno a la aplicación de la teoría de portafolio en la optimización del proceso de comercialización de energía continúan en auge. Un trabajo de reciente publicación es el desarrollado por Nanpeng, Tesfatsion y Chen-Ching [15]. Estos autores estudiaron el proceso de negociación de contratos bilaterales de electricidad, a la luz de la teoría de equilibrio de Nash, con el fin de identificar los potenciales resultados del proceso de negociación ante la incertidumbre que se presenta en las variables de mercado. 


\section{investigación}

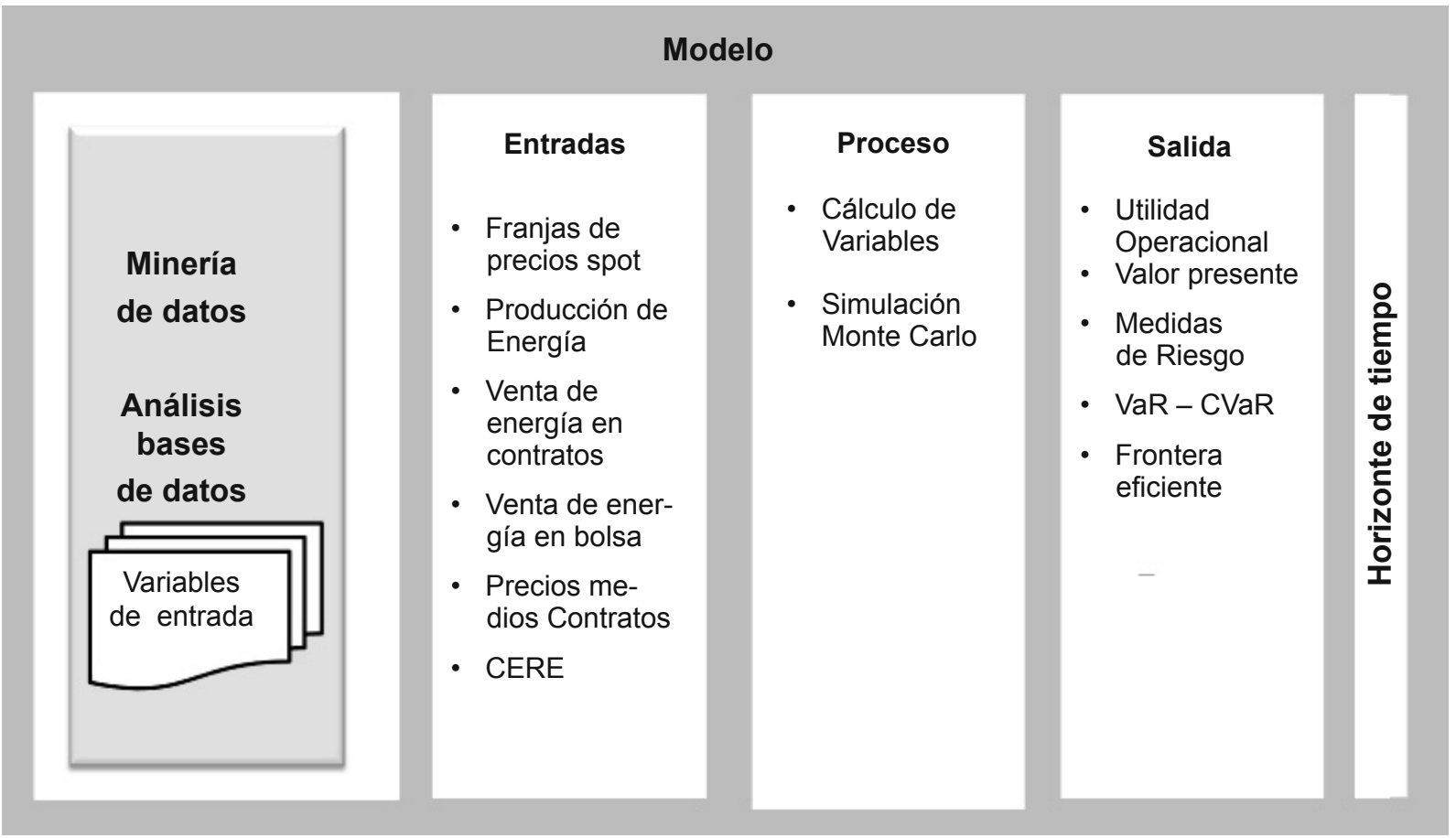

Figura 1. Estructura general del modelo de simulación

Fuente: elaboración propia.

Teniendo en cuenta los antecedentes presentados, en este trabajo se propone un modelo de valoración de riesgo en las transacciones de venta de energía mediante contratos a largo plazo, para un generador de energía en Colombia. El tema se aborda haciendo un análisis del sector eléctrico e identificando las variables determinantes, con las cuales se puede argumentar cuál es el punto óptimo de contratación que permitirá al generador maximizar el margen de beneficio y, a la vez, minimizar el riesgo de pérdida de valor.

\section{METODOLOGÍA}

El trabajo desarrollado se realizó en 3 fases. Las fases 1 y 2 corresponden al análisis de información relevante tanto en el mercado mayorista colombiano como a nivel de la compañía de generación; en la fase 3 se desarrolló el módulo de simulación de escenarios y definición de la fron- tera eficiente para la actividad de comercialización. Resultado de este proceso, se configuró un modelo de simulación cuyas variables de entrada fueron las siguientes: precios spot de la energía, producción de energía, venta de energía en contratos, precios medios de contratos y CERE (Costo Equivalente Real de Energía) (figura 1). Para determinar el comportamiento de las variables de entrada se realizó un análisis histórico de los valores de dichas variables en el contexto nacional, a partir de la base de datos administrada por el operador de mercado mayorista.

\subsection{Análisis descriptivo-series de datos históricos}

En la tabla 1 se presentan las series de datos históricas consideradas en el modelo, las cuales fueron obtenidas del sistema NEON disponible en la página de la compañía Expertos en Mercados 


\section{investigación}

Tabla 1. Series de datos históricos

\begin{tabular}{|c|c|c|c|c|}
\hline Nombre de la serie & Periodo histórico & Frecuencia & Datos & Unidad \\
\hline Precios spot de la bolsa de energía & \multirow{4}{*}{$\begin{array}{c}1 \text { Mar } 2001 \\
31 \text { Dic } 2011\end{array}$} & Diaria & 3,958 & $\$ / \mathrm{kWh}$ \\
\hline Generación real de 9 plantas & & Diaria & 32,073 & kWh \\
\hline Precios medios de contratos & & Diaria & 3,958 & $\$ / \mathrm{kWh}$ \\
\hline Precios medios costos CERE & & Mensual & 130 & $\$ / \mathrm{kWh}$ \\
\hline
\end{tabular}

Fuente: elaboración propia.

S.A.E.S.P. y estudiadas mediante el paquete estadístico Minitab 16.1.0.

Para el análisis de las series de los precios spot de la Bolsa de Energía Nacional, la serie de la generación real de las plantas y la serie de los precios medios de contratos, se estudió la serie diaria con la cual se determinó la serie ponderada mensual para cada una de las variables, capturando así la estacionalidad presente en el mercado.
En la figura 2 se presenta la serie histórica completa hasta el 31 de diciembre de 2011 desde que surgió la Bolsa de Energía en Colombia a partir del 20 de julio de 1995. En la figura 2 se pueden apreciar algunas de las causas de los picos y cambios abruptos de la serie. Esta serie presenta un patrón muy complejo debido a la estacionalidad múltiple (diaria, semanal, mensual y anual, inclusive). En cuanto a la estacionalidad se refleja claramente la fuerte volatilidad.

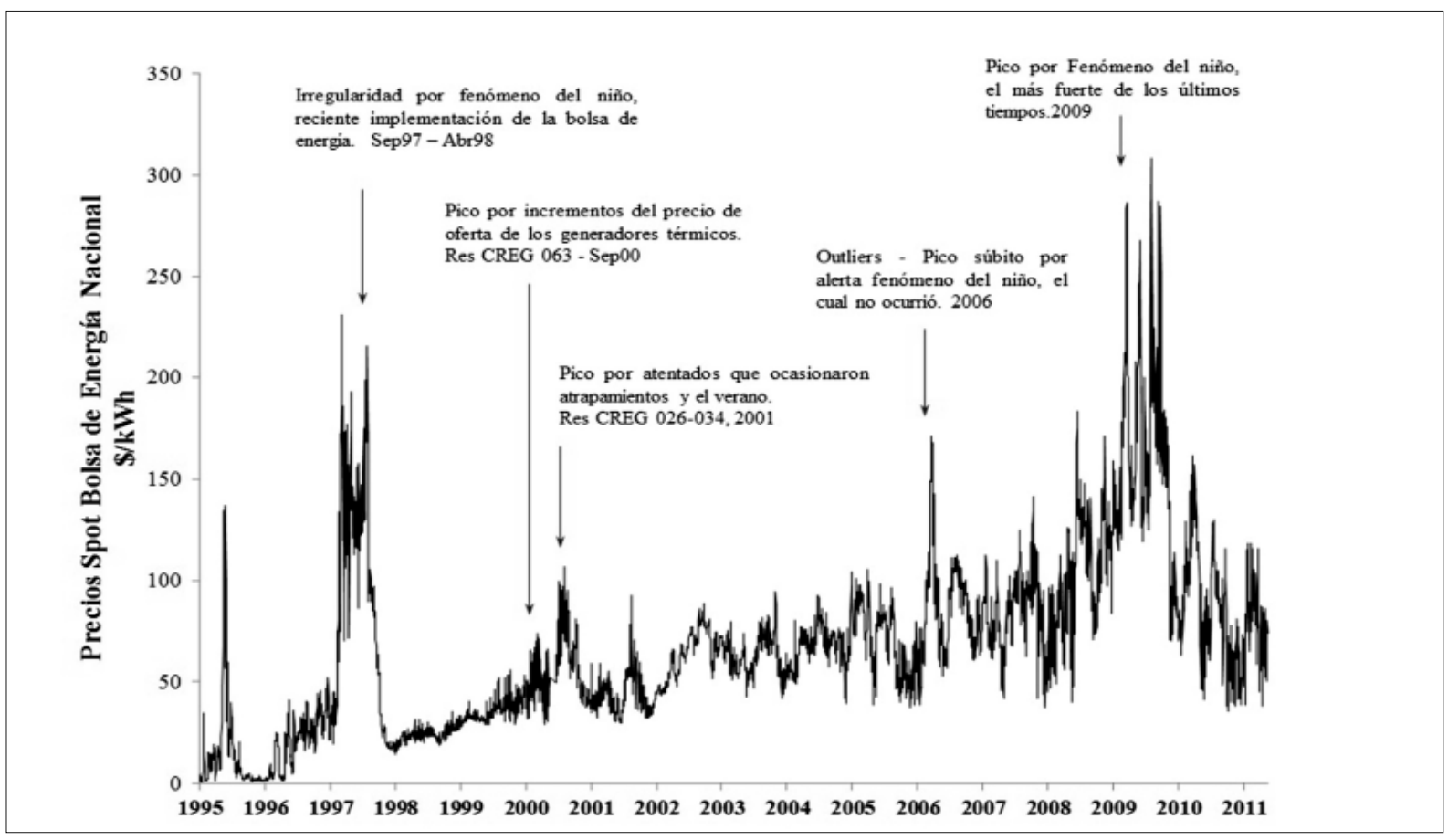

Figura 2. Serie histórica de la Bolsa de Energía Nacional

Fuente: El Tiempo, 16 de noviembre de 2011 


\section{investigación}

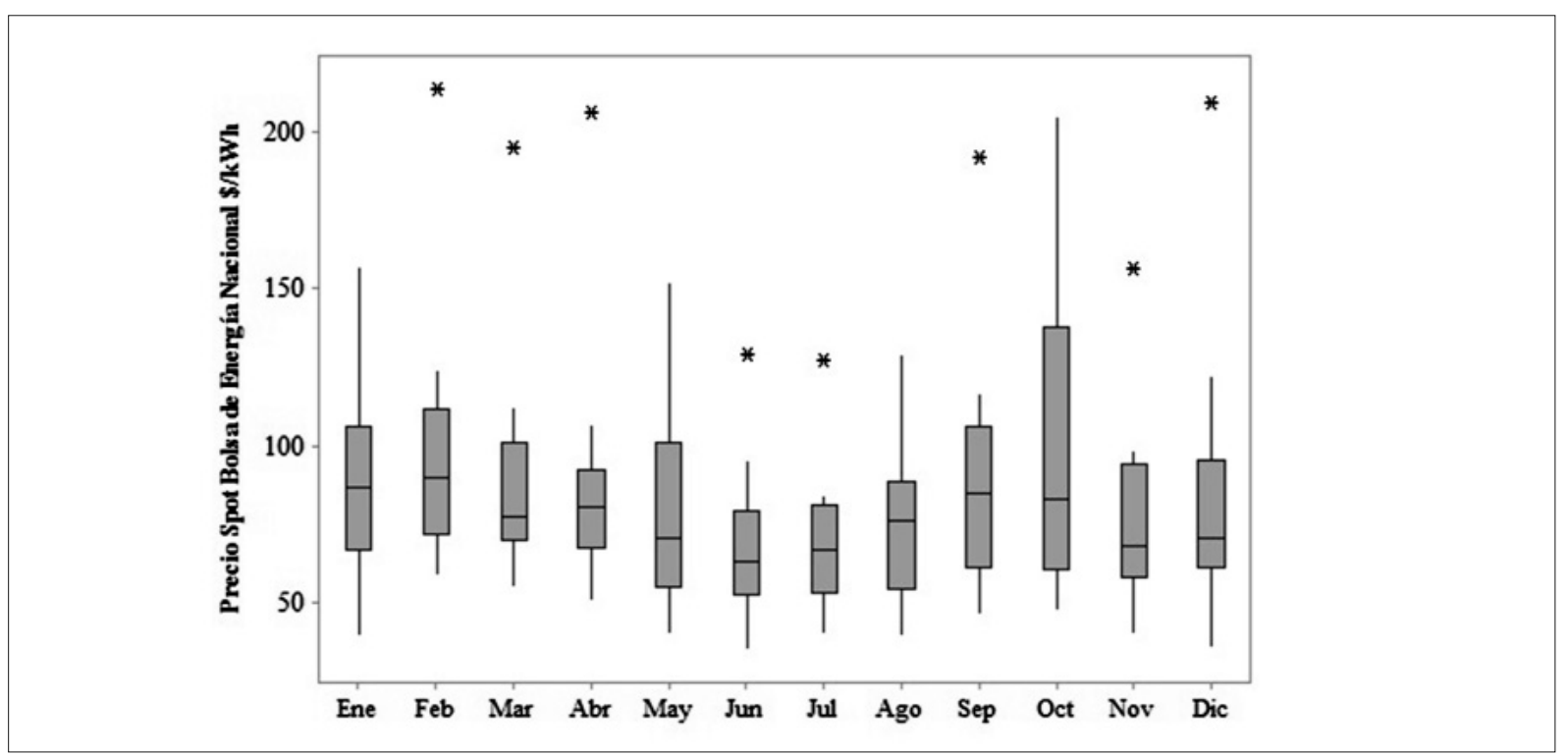

Figura 3. Comportamiento de la serie de precios spot mensuales agregada por mes

Fuente: elaboración propia.

La simple inspección visual de la serie parece indicar que el comportamiento de la volatilidad en el mercado es cambiante en el tiempo y sugiere la existencia de volatilidad agrupada. Como se observa en el comportamiento del precio a lo largo del periodo, son muy marcados los cambios de precio en diferentes momentos del horizonte. El precio muestra en su comportamiento los efectos complejos de la actividad de oferta, demanda, la regulación y la hidrología.

Considerando que la Comisión de Regulación de Energía y Gas (CREG) reguló los precios de energía mediante la resolución CREG-026 del 24 de febrero de 2001, en la cual se estableció una única oferta de precios diaria según despacho económico horario, se tomó la decisión de realizar el análisis estadístico descriptivo teniendo en cuenta los datos solo a partir del 1 de marzo de 2001 hasta el 31 de diciembre de 2011; además, para la ejecución de las pruebas, se utilizan los datos del periodo comprendido entre el 1 de enero de 2009 y el 31 de diciembre de 2011.
En la figura 3 se presenta el diagrama de cajas y alambres de la serie mensual de precios spot de la Bolsa de Energía Nacional que representa el comportamiento agregado por cada mes desde el 2001 hasta el 2011.

En esta representación se observa que históricamente el mes con mayor variabilidad de precios es octubre, cuando se reflejan precios ponderados mensuales hasta de $205 \$ / \mathrm{kWh}$ aproximadamente. Este mes presenta los precios más altos que se encuentran marcados por el cuartil 2, a diferencia de julio que tiene poca variabilidad y presenta un valor atípico dentro del mismo mes, ubicado por debajo de los puntos atípicos presentados en los demás meses. La observación atípica más alta se presentó en febrero. De acuerdo con el análisis de la serie, la causa de las observaciones atípicas obedece en gran medida a los altos precios presentados en los años 2009 y 2010.

En la figura 4 se presenta la serie histórica agregada por año, donde se observa que el año con mayor variabilidad de precios es el 2010, lo cual 


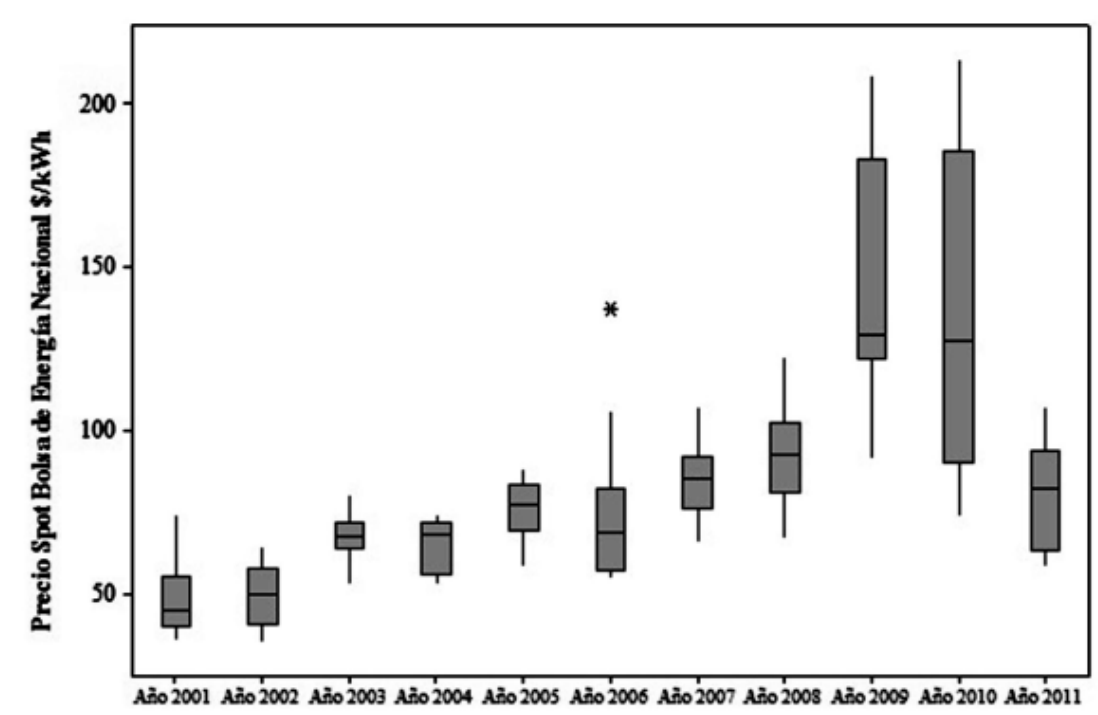

Figura 4. Comportamiento de la serie de precios spot mensuales agregada por año

Fuente: elaboración propia.

se explica por el fuerte verano a causa del efecto Fenómeno del Niño, que ocasionó precios muy altos desde el 2009. Por otro lado, el año de menor variabilidad fue el 2003, el cual presenta una concentración entre 50 y 80 \$/kWh aproximadamente.

En la tabla 2 se presenta el resumen de indicadores estadísticos descriptivos de la serie de los precios spot de la Bolsa de Energía Nacional tanto diarios como mensuales. Por otra parte, en la tabla 3 se muestran los principales indicadores estadísticos de la serie pero agregados por mes.
Si se considera el comportamiento de la serie y su complejidad para aplicar un método de pronóstico, en el presente trabajo se optó por la técnica de simulación de escenarios de acuerdo con las franjas de precios clasificadas, las cuales recogen el comportamiento histórico de la serie y el comportamiento agregado por mes.

En la figura 5 se presentan los intervalos en los cuales se simuló la distribución de probabilidad triangular para los precios spot de la Bolsa de Energía, para cada mes, de acuerdo con la franja de precios definida teniendo en cuenta el

Tabla 2. Indicadores estadísticos serie diaria y mensual de precios

\begin{tabular}{|c|c|c|c|c|c|c|c|c|}
\hline \multicolumn{8}{|c|}{ Estadística descriptiva de la serie diaria de los precios spot } \\
\hline N & Media & Desv.Est. & CoefVar & Mínimo & Q1 & Mediana & Q3 & Máximo \\
\hline 3927 & 82.20 & 37.80 & 45.98 & 29.62 & 58.82 & 73.15 & 94.39 & 308.07 \\
\hline \multicolumn{7}{|c|}{ Estadística descriptiva de la serie mensual de los precios spot } \\
\hline N & Media & Desv.Est. & CoefVar & Mínimo & Q1 & Mediana & Q3 & Máximo \\
\hline 129 & 84.21 & 36.76 & 43.66 & 35.39 & 60.91 & 74.70 & 94.16 & 213.57 \\
\hline
\end{tabular}

Fuente: elaboración propia. 


\section{investigación}

Tabla 3. Estadística descriptiva de la serie mensual de precios spot agregada por mes

\begin{tabular}{|c|c|c|c|c|c|c|c|c|c|}
\hline Mes & $\mathrm{N}$ & Media & Desv.Est. & CoefVar & Mínimo & Q1 & Mediana & Q3 & Máximo \\
\hline Ene & 10 & 90,6 & 34,3 & 37,8 & 39,9 & 67,0 & 87,0 & 105,9 & 157,1 \\
\hline Feb & 10 & 100,9 & 44,8 & 44,4 & 58,7 & 72,0 & 90,0 & 111,5 & 213,6 \\
\hline Mar & 11 & 91,1 & 38,0 & 41,8 & 55,1 & 69,8 & 77,5 & 101,1 & 195,2 \\
\hline Abr & 11 & 88,7 & 42,2 & 47,6 & 50,8 & 67,7 & 80,5 & 92,3 & 206,1 \\
\hline May & 11 & 79,3 & 33,1 & 41,7 & 40,1 & 55,4 & 70,9 & 101,1 & 151,9 \\
\hline Jun & 11 & 68,8 & 26,2 & 38,1 & 35,4 & 52,6 & 63,2 & 79,4 & 129,1 \\
\hline Jul & 11 & 69,5 & 24,2 & 34,9 & 40,4 & 53,3 & 66,8 & 81,1 & 127,3 \\
\hline Ago & 11 & 75,9 & 25,4 & 33,5 & 39,6 & 54,4 & 76,3 & 88,6 & 129,4 \\
\hline Sep & 11 & 88,9 & 40,9 & 46,0 & 46,3 & 61,4 & 85,2 & 106,0 & 192,1 \\
\hline Oct & 11 & 95,6 & 46,8 & 48,9 & 47,5 & 60,7 & 83,1 & 137,6 & 205,1 \\
\hline Nov & 11 & 77,6 & 31,3 & 40,4 & 40,0 & 58,5 & 68,1 & 94,2 & 156,5 \\
\hline Dic & 11 & 86,0 & 48,8 & 56,8 & 35,9 & 61,5 & 70,9 & 95,4 & 209,1 \\
\hline
\end{tabular}

Fuente: elaboración propia.

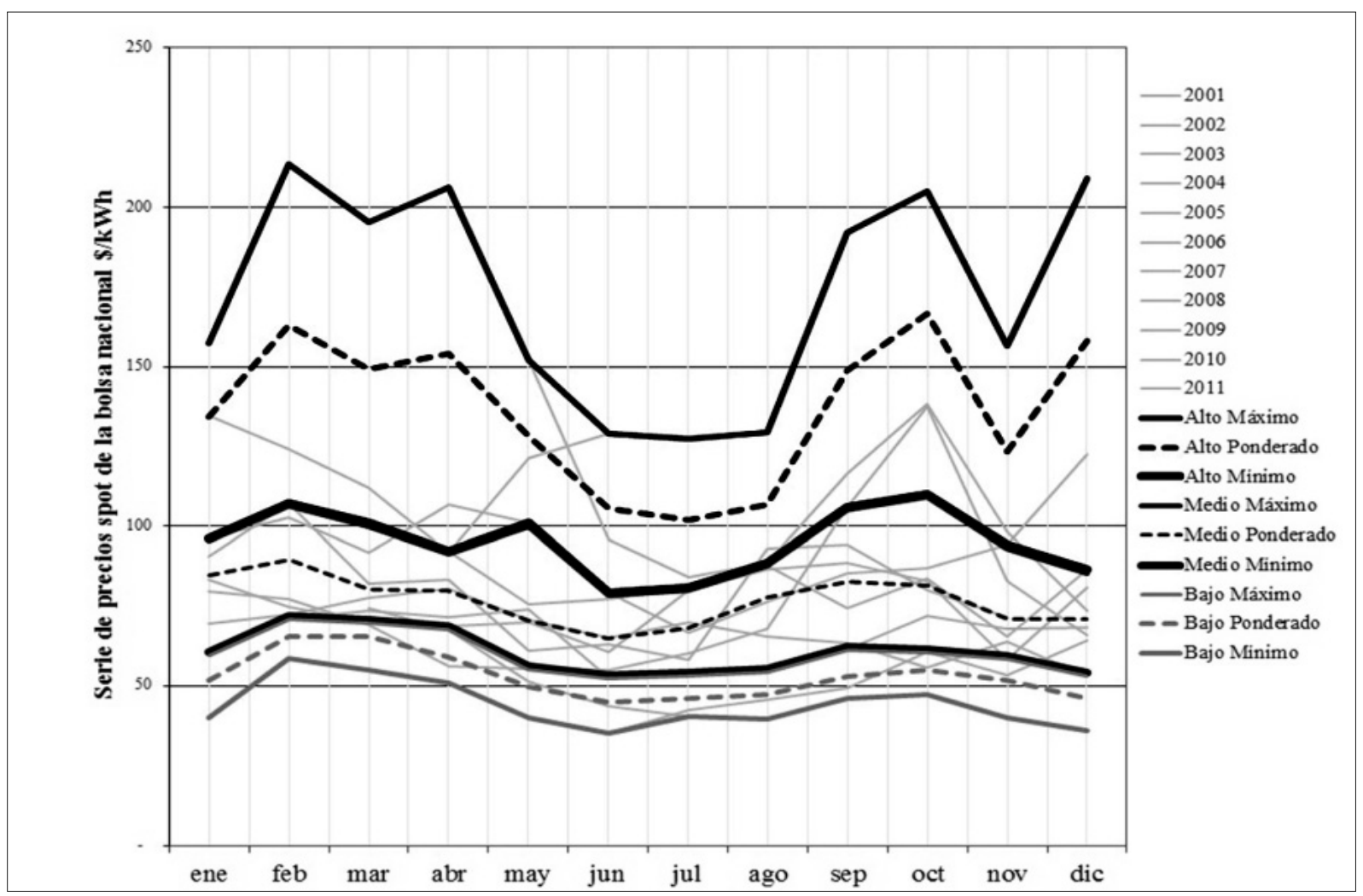

Figura 5. Serie histórica de los precios spot vs. las franjas de precios

Fuente: elaboración propia.

118 Tecnura | Vol. $18 \mid$ No. 39 | enero - marzo de 2014 


\begin{tabular}{|llrrrrrrrrrrrr|}
\hline Franja & & ene & feb & mar & abr & may & jun & jul & ago & sep & oct & nov & dic \\
\hline \multirow{3}{*}{ Alto } & Máximo & 157 & 214 & 195 & 206 & 152 & 129 & 127 & 129 & 192 & 205 & 156 & 209 \\
\cline { 2 - 13 } & Ponderado & 134 & 163 & 149 & 154 & 128 & 106 & 102 & 107 & 149 & 167 & 123 & 158 \\
& Minimo & 96 & 107 & 101 & 92 & 101 & 79 & 81 & 89 & 106 & 110 & 94 & 86 \\
\hline & Máximo & 95 & 106 & 100 & 91 & 100 & 78 & 80 & 88 & 105 & 109 & 93 & 85 \\
\hline \multirow{2}{*}{ Medio } & Ponderado & 85 & 89 & 80 & 80 & 71 & 65 & 68 & 78 & 83 & 82 & 71 & 71 \\
\hline & Minimo & 61 & 72 & 71 & 69 & 56 & 54 & 54 & 55 & 62 & 62 & 59 & 54 \\
\hline & Máximo & 60 & 71 & 70 & 68 & 55 & 53 & 53 & 54 & 61 & 61 & 58 & 53 \\
\hline \multirow{2}{*}{ Bajo } & Ponderado & 52 & 66 & 65 & 59 & 50 & 45 & 46 & 47 & 53 & 55 & 52 & 46 \\
\hline & Mínimo & 40 & 59 & 55 & 51 & 40 & 35 & 40 & 40 & 46 & 48 & 40 & 36 \\
\hline
\end{tabular}

Figura 6. Franjas de precios spot por mes

Fuente: elaboración propia.

\begin{tabular}{|c|c|c|c|c|c|c|c|c|c|c|c|c|}
\hline Año & ene & feb & mar & abr & may & jun & jul & ago & sep & oct & nov & dic \\
\hline 2001 & & & 74 & 68 & 51 & 44 & 40 & 40 & 46 & 48 & 40 & 36 \\
\hline 2002 & 40 & 59 & 55 & 51 & 40 & 35 & 43 & 46 & 49 & 61 & 53 & 64 \\
\hline 2003 & 70 & 72 & 77 & 80 & 71 & 65 & 70 & 66 & 64 & 56 & 64 & 53 \\
\hline 2004 & 60 & 71 & 73 & 72 & 74 & 53 & 53 & 54 & 61 & 72 & 68 & 68 \\
\hline 2005 & 83 & 75 & 70 & 69 & 70 & 60 & 80 & 87 & 88 & 83 & 58 & 81 \\
\hline 2006 & 80 & 77 & 69 & 56 & 55 & 55 & 60 & 68 & 106 & 138 & 83 & 66 \\
\hline 2007 & 90 & 107 & 101 & 92 & 76 & 77 & 81 & 88 & 75 & 84 & 65 & 86 \\
\hline 2008 & 96 & 103 & 92 & 107 & 101 & 79 & 67 & 76 & 85 & 87 & 94 & 122 \\
\hline 2009 & 135 & 124 & 112 & 92. & 121 & 129 & 127 & 129 & 192 & 205 & 156 & 209 \\
\hline 2010 & 157 & 214 & 195 & 206 & 152 & 96 & 84 & 89 & 116 & 138 & 98 & 73 \\
\hline 2011 & 95 & 107 & 82 & 83 & 61 & 63 & 58 & 93 & 94 & 80 & 72 & 58 \\
\hline
\end{tabular}

Figura 7. Franjas de precios spot por mes

Fuente: elaboración propia.

comportamiento histórico del precio ponderado para cada uno de los meses, en cada uno de los años estudiados.

En la figura 6 se muestran las franjas definidas para cada uno de los meses de enero a diciembre, en relación con los precios históricos observados.

De acuerdo con las franjas establecidas, para efecto de pruebas, a continuación se presentan los precios spot de la bolsa de energía en forma- to mensual según su comportamiento y considerando la franja en que debe ser simulado.

Además de la serie de precios spot de la Bolsa de Energía Nacional, se analizaron las series de generación real para cada una de las plantas dela compañía de generación objeto de estudio, en total 8 plantas de generación hidroeléctrica y una planta de generación termoeléctrica (tabla 4); adicionalmente, se analizó la serie de precios medios de contratos bilaterales y la serie de los costos CERE (tabla 5). 


\section{investigación}

Tabla 4. Estadística descriptiva de la serie mensual de la generación real por planta (KWh)

\begin{tabular}{|c|c|c|c|c|c|c|c|c|}
\hline Variable & $\mathrm{N}$ & Media & Desv.Est. & Mínimo & $\mathrm{Q} 1$ & Mediana & Q3 & Máximo \\
\hline Planta1 & 4,017 & $4,843,501$ & $3,243,643$ & 0 & $1,640,898$ & $4,878,949$ & $7,632,345$ & $10,448,984$ \\
\hline Planta2 & 4,017 & $2,857,853$ & $1,557,739$ & 0 & $1,618,142$ & $2,373,720$ & $3,891,374$ & $6,921,931$ \\
\hline Planta3 & 4,017 & 514,921 & 950,697 & 0 & 0 & 0 & 358,276 & $3,213,659$ \\
\hline Planta4 & 4,017 & 499,551 & 445,025 & 0 & 36,305 & 423,245 & 916,397 & $1,345,447$ \\
\hline Planta5 & 4,017 & 95,626 & 34,331 & 0 & 95,592 & 97,600 & 122,734 & 140,888 \\
\hline Planta6 & 4,017 & 98,646 & 40,558 & 0 & 68,836 & 95,779 & 131,672 & 187,560 \\
\hline Planta7 & 4,017 & 34,743 & 10,578 & 0 & 25,850 & 35,675 & 44,565 & 145,520 \\
\hline Planta8 & 409 & 251,186 & 119,751 & 0 & 175,806 & 252,198 & 340,981 & 450,853 \\
\hline Planta9 & 4,017 & 406,490 & $1,117,100$ & 0 & 0 & 0 & 0 & $4,873,155$ \\
\hline
\end{tabular}

Fuente: elaboración propia.

Tabla 5. Indicadores estadísticos series de precios diarios y mensuales de contratos y serie mensual de CERE

\begin{tabular}{|c|c|c|c|c|c|c|c|c|}
\hline \multicolumn{9}{|c|}{ Serie diaria de precios medios de contratos } \\
\hline $\mathrm{N}$ & Media & Desv.Est. & CoefVar & Mínimo & Q1 & Mediana & Q3 & Máximo \\
\hline 3958 & 82.57 & 19.54 & 23.66 & 51.54 & 71.02 & 73.84 & 103.45 & 122.95 \\
\hline \multicolumn{9}{|c|}{ Serie mensual de precios de contratos } \\
\hline $\mathrm{N}$ & Media & Desv.Est. & CoefVar & Mínimo & Q1 & Mediana & Q3 & Máximo \\
\hline 130 & 82.61 & 19.62 & 23.75 & 52.38 & 70.72 & 73.74 & 103.45 & 122.84 \\
\hline \multicolumn{9}{|c|}{ Serie mensual del CERE } \\
\hline $\mathrm{N}$ & Media & Desv.Est. & CoefVar & Mínimo & Q1 & Mediana & Q3 & Máximo \\
\hline 130 & 27.86 & 2.85 & 10.21 & 22.35 & 25.75 & 27.71 & 29.68 & 36.49 \\
\hline
\end{tabular}

Fuente: elaboración propia.

\subsection{Modelo de simulación}

A continuación se presentan los principales parámetros y variables del modelo de simulación desarrollado a partir de la información estadística previamente considerada (tablas 6 a 10).
Tabla 6. Índices del modelo

\begin{tabular}{|c|l|c|}
\hline Parámetro & \multicolumn{1}{|c|}{ Descripción } & \multicolumn{1}{c|}{ Unidad } \\
\hline$T$ & Índice de tiempo analizado & $t=1, \ldots, T$ \\
\hline$Z$ & $\begin{array}{l}\text { Índice de planta de generación de } \\
\text { energía eléctrica }\end{array}$ & $Z=1, \ldots, Z$ \\
\hline$W$ & Índice de escenarios & $W=1, \ldots, W$ \\
\hline
\end{tabular}

Fuente: elaboración propia. 
Tabla 7. Variables de decisión

\begin{tabular}{|c|l|c|c|}
\hline Parámetro & \multicolumn{1}{|c|}{ Descripción } & Unidad & Tipo \\
\hline$\%$ VEEC: & $\begin{array}{l}\text { Venta de energía estimada en contratos a largo plazo, se asume cons- } \\
\text { tante durante el tiempo analizado }\end{array}$ & Determinístico \\
\hline$\%$ VEEB: & $\begin{array}{l}\text { Venta de energía estimada en la bolsa de energía eléctrica, se asume } \\
\text { constante durante el tiempo analizado }\end{array}$ & $\%$ & Determinístico \\
\hline WACC: & $\begin{array}{l}\text { Costo promedio de capital - (WACC Weighted Average Cost of Capital), } \\
\text { se asume constante durante el tiempo analizado }\end{array}$ & Determinístico \\
\hline$\% G O V:$ & $\begin{array}{l}\text { Gastos operacionales de venta, se asume constante durante el tiempo } \\
\text { analizado }\end{array}$ & $\begin{array}{l}\text { Gastos operacionales de administración, se asume constante durante el } \\
\text { tiempo analizado }\end{array}$ & Determinístico \\
\hline
\end{tabular}

Fuente: elaboración propia.

Tabla 8. Variables del entorno

\begin{tabular}{|c|l|c|c|}
\hline Parámetro & \multicolumn{1}{|c|}{ Descripción } & Unidad & Tipo \\
\hline PEB $_{t w}:$ & $\begin{array}{l}\text { Precios de energía eléctrica en bolsa estimado, en un periodo t de un } \\
\text { escenario } w\end{array}$ & COP/\$kWh & Estocástico \\
\hline PEP $_{t z}:$ & $\begin{array}{l}\text { Producción de energía eléctrica estimada, en un periodo t, de una planta } \\
\text { de generación de energía eléctrica } z\end{array}$ & GWh & Estocástico \\
\hline PEC $_{t}:$ & $\begin{array}{l}\text { Precio de energía estimado de contratos de energía eléctrica, en un } \\
\text { periodo } t\end{array}$ & COP/\$kWh & Estocástico \\
\hline $\mathrm{CCR}_{t}:$ & Costos CERE estimado, en un periodo $t$ & COP/\$kWh & Estocástico \\
\hline TIR: & $\begin{array}{l}\text { Tasa de impuesto de renta, se asume constante durante el tiempo } \\
\text { analizado }\end{array}$ & Determinístico \\
\hline
\end{tabular}

Fuente: elaboración propia.

Tabla 9. Variables de proceso

\begin{tabular}{|c|c|c|c|}
\hline Parámetro & Descripción & Unidad & Tipo \\
\hline $\mathrm{VEC}_{t}:$ & Venta de energía estimada en contratos a largo plazo, en un periodo $t$ & $\mathrm{kWh}$ & Estocástico \\
\hline $\mathrm{VEB}_{t}:$ & $\begin{array}{l}\text { Venta de energía estimada en la bolsa de energía eléctrica, en un perio- } \\
\text { do t }\end{array}$ & kWh & Estocástico \\
\hline $\mathrm{CCRC}_{t}:$ & $\begin{array}{l}\text { Costos CERE estimados de la energía vendida en contrato, en un perio- } \\
\text { do } t\end{array}$ & $\mathrm{COP} / \$ \mathrm{kWh}$ & Estocástico \\
\hline $\mathrm{CCRB}_{t}$ & Costos CERE estimados de la energía vendida en bolsa, en un periodo $t$ & $\mathrm{COP} / \$ \mathrm{kWh}$ & Estocástico \\
\hline
\end{tabular}

Fuente: elaboración propia. 


\section{investigación}

Tabla 10. Variables de salida

\begin{tabular}{|c|c|c|c|}
\hline Parámetro & Descripción & Unidad & Tipo \\
\hline IOVEC $_{t}$ & $\begin{array}{l}\text { Ingresos operacionales por venta de energía estimada en contratos a } \\
\text { largo plazo, en un periodo } t\end{array}$ & COP & Estocástico \\
\hline IOVEB: & $\begin{array}{l}\text { Ingresos operacionales por venta de energía estimada en la bolsa de } \\
\text { energía eléctrica, en un periodo t }\end{array}$ & COP & Estocástico \\
\hline $\mathrm{CCRC}_{t}$ & $\begin{array}{l}\text { Costos CERE estimados de la energía vendida en contrato, en un perio- } \\
\text { do } t\end{array}$ & COP & Estocástico \\
\hline $\mathrm{CCRB}_{t}:$ & Costos CERE estimados de la energía vendida en bolsa, en un periodo $t$ & $\mathrm{COP}$ & Estocástico \\
\hline $\mathrm{UBT}_{t}$ & Utilidad bruta, en un periodo $t$ & COP & Estocástico \\
\hline $\mathrm{MC}_{t}$ & Margen de contribución, en un periodo t & $\%$ & Estocástico \\
\hline $\mathrm{GOV}_{t}$ & Gastos operacionales de venta, en un periodo $t$ & COP & Determinístico \\
\hline $\mathrm{GOA}_{t}:$ & Gastos operacionales de administración, en un periodo $t$ & COP & Determinístico \\
\hline $\mathrm{UO}_{\mathrm{t}}$ & Utilidad operacional, en un periodo $t$ & COP & Estocástico \\
\hline $\mathrm{MUO}_{t}$ & Margen de utilidad operacional, en un periodo $t$ & $\%$ & Estocástico \\
\hline UOVEC $_{t}$ & $\begin{array}{l}\text { Utilidad operacional por venta de energía estimada en contratos a largo } \\
\text { plazo, en un periodo } t\end{array}$ & COP & Estocástico \\
\hline MUOVEC $_{t}$ & $\begin{array}{l}\text { Margen de utilidad operacional por venta de energía estimada en contra- } \\
\text { tos a largo plazo, en un periodo } t\end{array}$ & $\%$ & Estocástico \\
\hline UOVEC: & $\begin{array}{l}\text { Utilidad operacional por venta de energía estimada en la bolsa de energía } \\
\text { eléctrica, en un periodo t }\end{array}$ & COP & Estocástico \\
\hline MUOVEB $_{t}$ & $\begin{array}{l}\text { Margen de utilidad operacional por venta de energía estimada en la bolsa } \\
\text { de energía eléctrica, en un periodo t }\end{array}$ & $\%$ & Estocástico \\
\hline VPN: & Valor presente neto de las utilidades del horizonte de tiempo de análisis & $\%$ & Estocástico \\
\hline
\end{tabular}

Fuente: elaboración propia.

La función de utilidad (ecuación 1) para el generador en un periodo $t$, está compuesta por los siguientes elementos:

- Ingresos operacionales por venta de energía estimada en contratos a largo plazo

- Ingresos operacionales por venta de energía estimada en la Bolsa de Energía Eléctrica

- Costos CERE por generación de energía eléctrica

- Gastos operacionales de venta

- Gastos operacionales de administración

$$
\begin{gathered}
U O=\sum_{t=1}^{T}(I O V E C+I O V E B)- \\
(C C R C+C C R B)-(G O V+G O A)
\end{gathered}
$$

A su vez, cada uno de los elementos que conforman la función de utilidad se determina de acuerdo con lo expresado en las ecuaciones (2) a (7):

$$
\begin{aligned}
& I_{\text {IOVEC }}=V E C_{t} \cdot P E C_{t} \\
& I O V E B_{t}=V E B_{t} \cdot P E B_{t} \\
& \operatorname{CCRC}_{t}=V E C_{t} \cdot C C R_{t} \\
& C C R B_{t}=V E B_{t} \cdot C C R_{t} \\
& G_{O}=\left(\left(I O V E C_{t} \cdot I_{t} O V E B_{t}\right) \cdot \% G O V\right)(6) \\
& G O A_{t}=\left(\left(I O V E C_{t} \cdot I O V E B_{t}\right) \cdot \% G O A\right)(7)
\end{aligned}
$$

Las restricciones consideradas para el modelo fueron las siguientes (ecuaciones (8) y (9)): 
Tabla 11. Escenarios simulados

\begin{tabular}{|c|c|c|}
\hline Escenario 1 & Escenario 2 & Escenario 3 \\
\hline $\begin{array}{l}\text { Los } 36 \text { periodos se simulan en } \\
\text { la franja de precios medios. }\end{array}$ & $\begin{array}{l}\text { De acuerdo con el comportamiento de precios } \\
\text { spot reales de los años } 2009,2010 \text { y } 2011 \text {, cada } \\
\text { periodo se simula de acuerdo con la franja de } \\
\text { precios correspondiente; en este caso el año } \\
2009 \text { y } 2010 \text { presentan gran parte de los perio- } \\
\text { dos en la franja de precios altos, el } 2011 \text { gran } \\
\text { parte en la franja de precios medios. }\end{array}$ & $\begin{array}{l}\text { Los periodos se simulan sin considerar } \\
\text { las franjas de precios, se trabaja con el } \\
\text { precio mínimo, promedio ponderado y el } \\
\text { máximo presentado en cada mes, según } \\
\text { la serie histórica. }\end{array}$ \\
\hline \multicolumn{3}{|c|}{$\begin{array}{l}\% \text { Participación en contratos: Se realizan } 21 \text { simulaciones, generando resultados del valor presente de la utilidad operacional } \\
\text { para distintas participaciones desde } 60 \% \text { hasta } 100 \% \text {, con cambio cada } 2 \% \text {. }\end{array}$} \\
\hline
\end{tabular}

Fuente: elaboración propia.

$$
\begin{gathered}
\text { Las } \% \text { VEEC }+\% \text { VEEB }=100 \% \\
70 \% \leq \% \text { VEEC } \leq 90 \% \\
\text { IOVEB,CCRC, CCRB, GOV , GOA }>0(10)
\end{gathered}
$$

La función objetivo utilizada en el modelo de optimización, complementario a la simulación, fue la maximización del valor presente de la utilidad operacional entendido esto como una de las componentes principales del flujo de caja de la compañía.

\section{RESULTADOS}

Los resultados obtenidos mediante la aplicación del modelo desarrollado se presentan de conformidad con los diferentes escenarios analizados (tabla 11).

En las figuras 8,9 y 10 se presentan los resultados obtenidos del modelo propuesto en los escenarios 1,2 y 3 respectivamente. Los resultados muestran el valor presente de la utilidad operacional en el horizonte de tiempo del modelo: tres años.

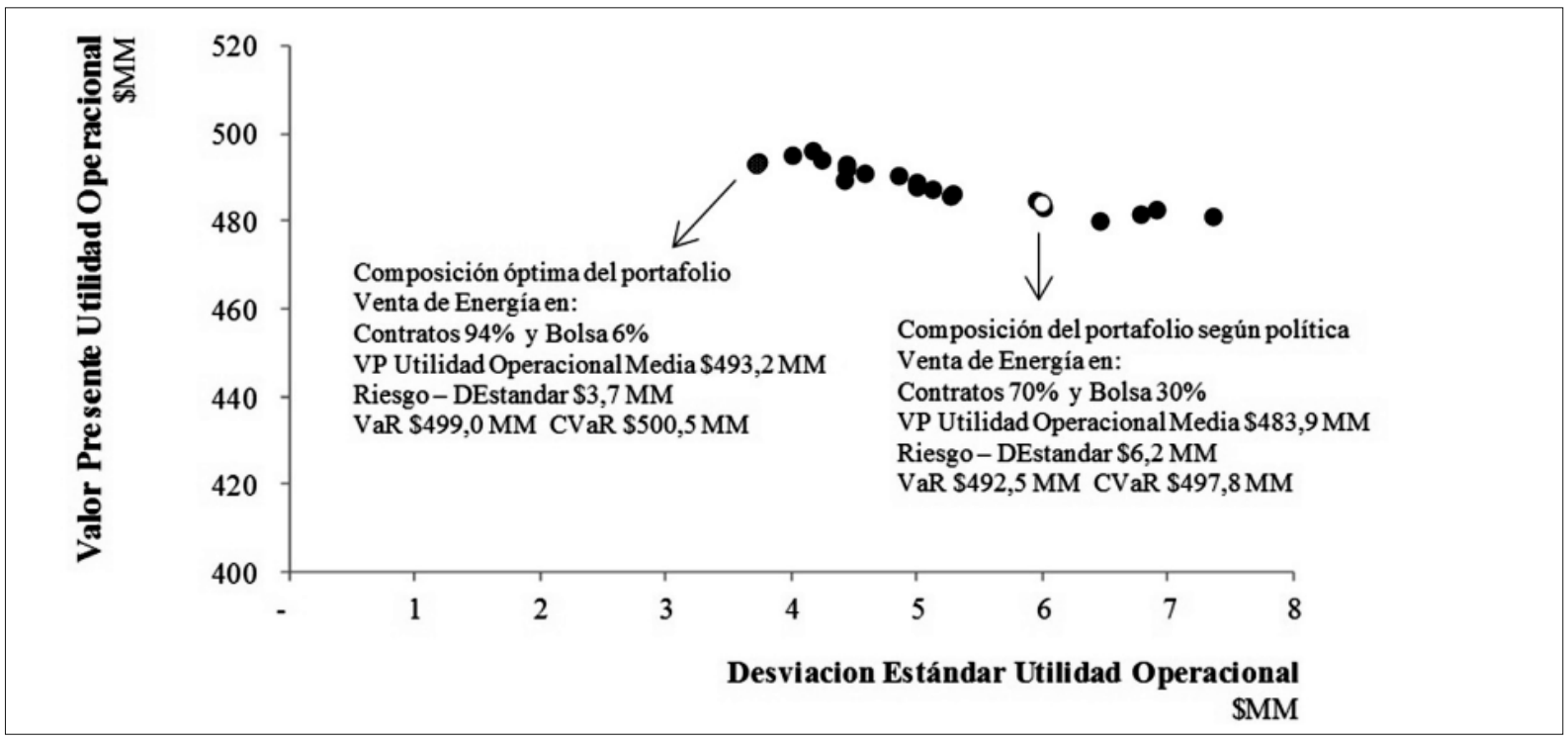

Figura 8. Resultado del valor presente de la utilidad operacional en el escenario 1

Fuente: elaboración propia. 


\section{investigación}

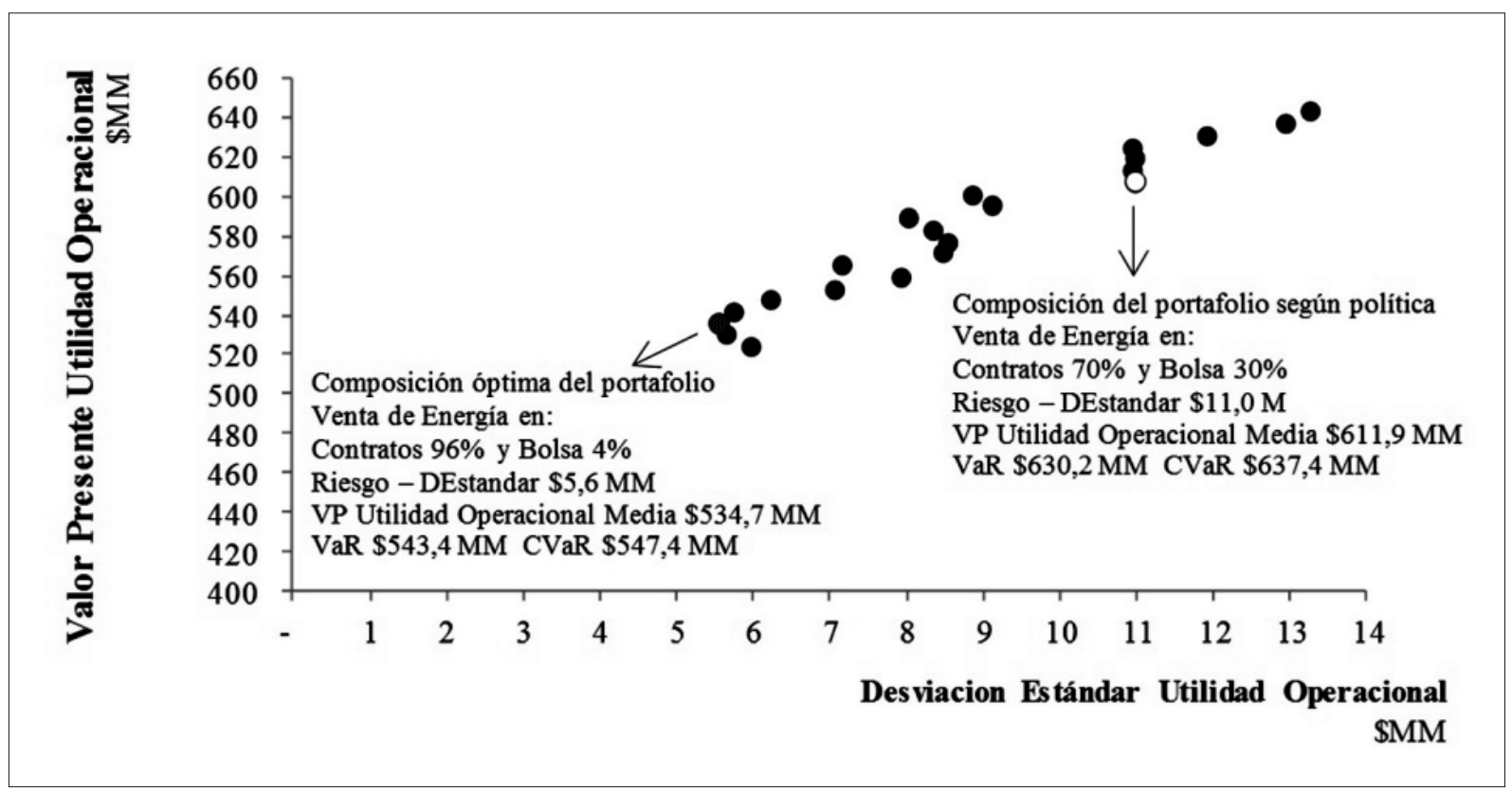

Figura 9. Resultado del valor presente de la utilidad operacional en el escenario 2

Fuente: elaboración propia.

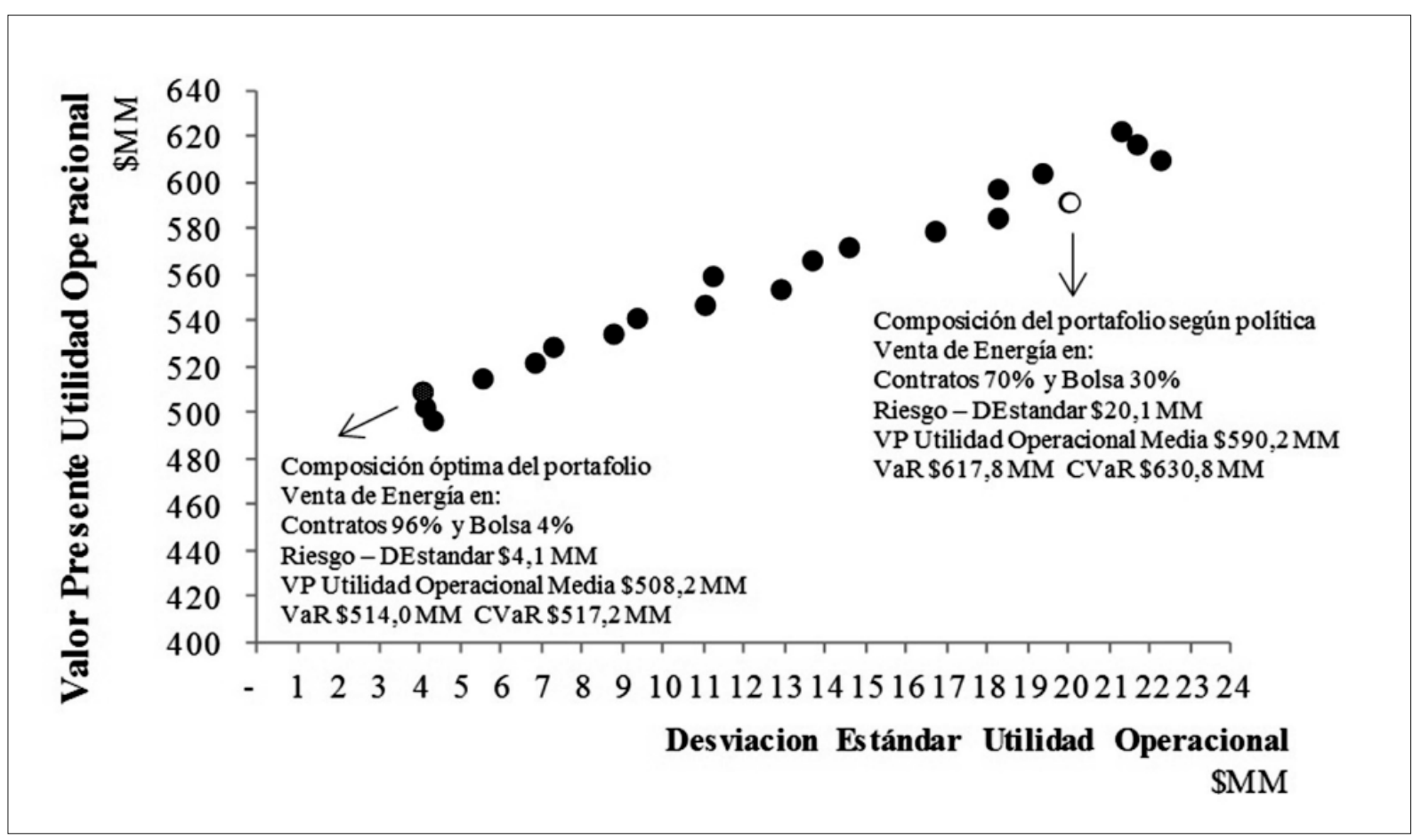

Figura 10. Resultado del valor presente de la utilidad operacional en el escenario 3

Fuente: elaboración propia. 


\section{investigación}

Tabla 12. Coeficiente de variación política 70-30 y punto de riesgo mínimo

\begin{tabular}{|l|c|c|c|}
\hline \multicolumn{1}{|c|}{ Coeficiente de variación (\%) } & Escenario 1 & Escenario 2 & Escenario 3 \\
\hline Política 70-30 & 1.28 & 1.80 & 3.41 \\
\hline Punto de riesgo mínimo & 0.75 & 1.05 & 0.81 \\
\hline
\end{tabular}

Fuente: elaboración propia.

Como se puede observar en cada una de las figuras correspondientes a los diferentes escenarios, la política corporativa definida por el generador en relación con el porcentaje de energía a comercializar vía contratos es de $70 \%$, y vía bolsa o mercado spot, de $30 \%$. El interrogante que subyace en este análisis es si la posición actual del generador corresponde al punto de contratación óptima en términos de riesgo y retorno. Dado que este es un análisis en términos de frontera eficiente, es indispensable conocer el nivel de aversión al riesgo del generador a fin de determinar dicha posición óptima de contratación. En la tabla $12 \mathrm{se}$ propone un análisis del coeficiente de variación para cada uno de los escenarios analizados, teniendo en cuenta los indicadores asociados tanto a la política corporativa (70-30) como al punto de riesgo mínimo en cada uno de los casos.

Como era de esperarse, moverse dentro de la frontera eficiente hacia escenarios de riesgo mínimo plantea coeficientes de variación menores. A diferencia de los resultados atípicos obtenidos en el escenario 1, donde el punto de riesgo mínimo presenta un mejor resultado en términos de valor presente de utilidades que el punto correspondiente a la política corporativa donde se asume un mayor nivel de riesgo, en los demás escenarios los resultados implican que el deseo de mayores niveles de utilidad implican la adopción de un mayor nivel de riesgo.

En las figuras 9 y 10 se puede observar que la definición empírica de política de contratación que ha establecido la empresa, si bien no se encuentra en el marco de la frontera eficiente, dado que para el mismo nivel de riesgo existen mejores puntos de rentabilidad, no se encuentra muy lejos de la política óptima para el nivel de riesgo asumido.

Al revisar los resultados obtenidos en los diferentes escenarios, el generador debe considerar su nivel de aversión al riesgo con el fin de definir si los niveles de utilidad marginal que puede llegar a obtener asumiendo posiciones más activas en bolsa compensan el nivel de riesgo marginal que se asume en dichas posiciones.

\section{CONCLUSIONES}

El modelo desarrollado reconoce las deficiencias que en materia de pronóstico de precios de energía se presentan con los modelos tradicionales. Los modelos tradicionales de pronóstico de precios, en el caso de activos tan volátiles como la electricidad, presentan un bajo nivel de confiabilidad en periodos como los que habitualmente se utilizan en la contratación bilateral de energía que pueden oscilar entre 1 y 3 años. Por este motivo, la valoración cuantitativa de los niveles de exposición al riesgo, medidos a través de técnicas como la simulación de Monte Carlo, constituye un apoyo adecuado en esta clase de procesos de toma de decisión.

En el presente trabajo se sugirió un método histórico alternativo, basado en la simulación Monte Carlo, teniendo en cuenta agrupamientos por franjas de precios en periodos similares. Aunque existen otras técnicas de estimación de precios basados en procesos estocásticos como las que se proponen en la literatura, el modelo planteado presenta la ventaja de facilitar la comprensión del 
fenómeno simulado a los funcionarios encargados del proceso de gestión del mercado mayorista de energía, ya que el objetivo, más que el pronóstico en sí mismo, es la valoración del nivel de riesgo que se asume cuando se adopta una determinada posición de contratación.

Una posible extensión del presente trabajo sería la consideración de modelos alternativos de estimación de las variables de entrada del modelo de simulación considerado, esto con el fin de establecer un marco de comparación frente al modelo empírico basado en simulación histórica que se propone en este artículo.

El análisis de frontera eficiente que se propone en el presente trabajo puede utilizarse para determinar políticas de contratación óptimas considerando el nivel de aversión al riesgo del agente decisor. Sin embargo, en esta clase de procesos de optimización no es posible hablar de un óptimo global, ya que el nivel de aversión al riesgo del decisor es determinante en términos del nivel de rentabilidad que se puede obtener.

\section{REFERENCIAS}

[1] XM, El mercado mayorista y su administración, Medellín: XM S.A., 2010.

[2] T.S. Chung, S.H. Zhang, C.W. Yu, and K.P. Wong, "Electricity market risk management using forward contracts with bilateral options", IEEE Proceedings: Generation, Transmission and Distribution, vol. 150, pp. 588-594, 2003.

[3] I. Herguera, "Bilateral contracts and the spot market for electricity: some observations on the British and the NordPool experiences", Utilities Policy, vol. 9, pp. 73-80, 2000 .

[4] L.G.B. Marzano, A.C.G. MeIo, and R.C. Souza, "An approach for portfolio optimization of energy contracts in the Brazilian electric sector", Power Tech Conference Proceedings, 2003 IEEE Bologna, vol. 3, p. 8, 2003.

[5] M. Carrion, A.B. Philpott, A.J. Conejo, and J.M. Arroyo, "A Stochastic Programming Approach to Electric Energy Procurement for Large Consumers", Power Systems,
IEEE Transactions on, vol. 22, pp. 744754, 2007.

[6] M. Liu and F.F. Wu, "Portfolio optimization in electricity markets",Electric Power Systems Research, vol. 77, pp. 1000-1009, 2007.

[7] S.E. Khatib and F.D. Galiana, "Negotiating Bilateral Contracts in Electricity Markets", Power Systems, IEEE Transactions on, vol. 22, pp. 553-562, 2007.

[8] N.M. Pindoriya, S.N. Singh, and S.K. Singh, "Optimal Generation Portfolio Allocation in Competitive Electricity Market", India Conference (INDICON), 2009 Annual IEEE, pp. 1-4, 2009.

[9] A.C. Nerves and L.M.D. Umali, "Risk assessment of energy trading for a generation company with bilateral contracts", in 2010 9th International Power and Energy Conference, IPEC 2010, 2010, pp. 1123-1128.

[10] R.G. Karandikar, S.A. Khaparde, and S.V. Kulkarni, "Strategic evaluation of bilateral 


\section{investigación}

contract for electricity retailer in restructured power market", International Journal of Electrical Power and Energy Systems, vol. 32, pp. 457-463, 2010.

[11] E. Delarue, C. De Jonghe, R. Belmans, and W. D'Haeseleer, “Applying portfolio theory to the electricity sector: Energy versus power", Energy Economics, vol. 33, pp. 12-23, 2011.

[12] H. Kebriaei, A. Rahimi-Kian, and V. J. Majd, "An agent-based system for bilateral contracts of energy",Expert Systems with Applications, vol. 38, pp. 11369-11376, 2011.

[13] D.J.R. Arias, F.D.B. Cardona, and S.I. Harold, "Contract price of a bilateral contract using risk assessment: With application to colombian wholesale electricity market", ANDESCON, 2010 IEEE, pp. 1-5, 2010.

[14] F. Gökgöz and M.E. Atmaca, "Financial optimization in the Turkish electricity market: Markowitz's mean-variance approach", Renewable and Sustainable Energy Reviews, vol. 16, pp. 357-368, 2012.

[15] Y. Nanpeng, L. Tesfatsion, and L. ChenChing, "Financial Bilateral Contract Negotiation in Wholesale Electricity Markets Using Nash Bargaining Theory", Power Systems, IEEE Transactions on, vol. 27, pp. 251-267, 2012. 